\title{
Scientific landscape of oxidative stress in male reproductive research: A scientometric study
}

\author{
Ashok Agarwal $^{\mathrm{a}, *}$, Saradha Baskaran ${ }^{\mathrm{a}}$, Manesh Kumar Panner Selvam ${ }^{\mathrm{a}}$, Renata Finelli ${ }^{\mathrm{a}}$, \\ Catalina Barbarosie $^{\mathrm{a}, \mathrm{b}}$, Kathy Amy Robert ${ }^{\mathrm{a}}$, Concetta Iovine ${ }^{\mathrm{a}}$, Kruyanshi Master ${ }^{\mathrm{a}}$, Ralf Henkel ${ }^{\mathrm{a}, \mathrm{c}}$ \\ ${ }^{a}$ American Center for Reproductive Medicine, Cleveland Clinic, Cleveland, $\mathrm{OH}, \mathrm{USA}$ \\ ${ }^{\mathrm{b}}$ Department of Genetics, Faculty of Biology, University of Bucharest, Bucharest, Romania \\ ${ }^{\mathrm{c}}$ Department of Medical Bioscience, University of the Western Cape, Bellville, South Africa
}

\section{A R T I C L E I N F O}

\section{Keywords:}

Diagnosis

Male infertility

Oxidative stress

Prognosis

Publications

Scientometric analysis

\begin{abstract}
A B S T R A C T
Unraveling the role of reactive oxygen species and associated oxidative stress (OS) in male reproduction is one of the key areas of male reproductive research. This article illustrates the scientific landscape of OS in male reproductive research over the past several decades (1941-2018) using a scientometric approach. Scientometric data (articles per year, authors, affiliations, journals, and countries) on OS related to male reproduction were retrieved from the Scopus database and analyzed for each decade. Our analysis revealed an increasing trend in OS-based male reproductive research from 1941 to 2018 with a steep raise in publications and research collaborations starting from the period $1991-2000\left(R^{2}=0.81\right)$. Semen abnormalities and varicocele were the major areas investigated in relation to OS with the highest positive trend in publications from the time interval 1981-1990 to 2011-2018. Analysis of publications based on OS assessment techniques revealed chemiluminescence $(n=180)$ and evaluation of antioxidants $(n=300)$ as the most widely used direct and indirect tests, respectively. Furthermore, prognostic/diagnostic studies on OS evaluation increased significantly over the time. Our analysis highlights the evolution of OS in male reproductive research and its emergence as an important prognostic and diagnostic tool in the evaluation of male infertility.
\end{abstract}

\section{Introduction}

Reactive oxygen species (ROS) are chemically highly reactive oxygen derivatives, some of which are free radicals possessing unpaired electrons in the outer orbit and therefore having an increased tendency to attract electrons from nearby molecules to attain stability [1]. At physiological levels, ROS act as signaling molecules in cell proliferation and survival, and thereby play an important role in the maintenance of homeostasis [2]. However, elevated levels of ROS induce oxidative damage leading to the impairment of cell structure and function [3]. Cells are equipped with antioxidants that counteract and regulate the levels of ROS by converting them to stable non-radical compounds and protect the cells from the deleterious effects of ROS [4]. Therefore, a balance between the ROS and antioxidants is pivotal for normal cell functions. When the production of ROS overweighs the scavenging capacity of antioxidants, a state of oxidative stress (OS) prevails [5]. OS has been associated with a myriad of pathologies including diabetes, cancer, neurodegeneration and infertility $[2,6]$.
Since the 1940s, scientists have carried out intense research to unravel the role of ROS in male reproduction. The reports of MacLeod (1943) and Tosic and Walton (1946) on the ability of spermatozoa to generate ROS were the first to indicate ROS production by living cells $[7,8]$. Subsequently, a series of publications by Jones et al. in the 1970s highlighting the susceptibility of spermatozoa to OS are landmark articles in the field of male reproductive research [9-12]. Further research focused on the impact of ROS on sperm functions in the mid1980 's and on the physiological role of ROS in the 1990's [13-15]. Studies reveal that low levels of ROS play an important role in various physiological processes such as sperm maturation, hyperactivation, acrosome reaction, capacitation, and fertilization [16,17]. Increased levels of ROS affect proteins, lipids, and DNA, causing lipid peroxidation and sperm DNA damage, which results in poor fertilizing ability [2]. Therefore, maintaining a subtle balance between ROS generation and elimination is crucial for normal sperm function and successful conception [17].

OS plays a pivotal role in the pathophysiology of male infertility

\footnotetext{
${ }^{*}$ Corresponding author. American Center for Reproductive Medicine, Cleveland Clinic, Mail Code X-11, 10681 Carnegie Avenue, Cleveland, OH, 44195, USA. E-mail address: agarwaa@ccf.org (A. Agarwal).

URL: http://www.Clevelandclinic.org/ReproductiveResearchCenter (A. Agarwal).
} 


\section{List of abbreviations}

DCFH-DA dichloro-dihydro-fluorescein-diacetate

ESR electron spin resonance

FITC fluorescein isothiocyanate

NBT nitroblue tetrazolium

ORP oxidation-reduction potential

MMP mitochondrial membrane potential

TAC total antioxidant capacity

8-OHdG 8-hydroxy-2'-deoxyguanosine

[18]. About $30 \%-40 \%$ of infertile men have increased levels of ROS in seminal plasma [19]. Recently, Agarwal et al. proposed the terminology Male Oxidative Stress Infertility (MOSI) for idiopathic infertile men suffering from OS [20]. Several reviews and meta-analyses have been published on OS and male infertility [21-24]. However, to date, there is no information available regarding the publication trends on OS in male reproductive research.

Scientometrics is a quantitative analysis of scientific literature, which reflects the growth of a specific research field [25]. It depicts the trend of one or more of the following: top countries, institutions, authors, and journals publishing scientific articles. Furthermore, co-authorship networks and institutional collaboration patterns can also be analyzed using scientometric data [26]. Until now, only few reports on the scientometric analysis of male infertility have been published $[27,28]$. Recently, we conducted an in-depth analysis of the publication trends in male infertility from 1998 to 2017 using the Funnel Model [29]. Subsequently, we focused on the publication trends of specific key topics related to male infertility such as proteomics and sperm DNA fragmentation (SDF) $[29,30]$. The results of the latter indicated an increased number of publications on OS as a key mechanism in mediating SDF [29]. These results intrigued us to investigate the publication trends and research directions of OS in male reproductive research.

Therefore, this study aimed to carry out a comprehensive, stepwise analysis of the scientific publications on OS related to male reproduction. This analysis would serve as a blueprint of the publication trends on OS in male reproductive research to provide a great source of information on current research, hot topics and widely used techniques as well as help scientists to establish the direction of future research.

\section{Materials and methods}

\subsection{Ethics statement}

This study is exempted from the approval of the Institutional Review Board (IRB) as it was conducted using the scientometric data retrieved from Scopus and did not involve any human subject.

\subsection{Data source}

The scientometric data was retrieved from Scopus as it is one of the most comprehensive bibliographic databases having over 70 million records and 1.4 billion cited references (https://www.elsevier.com/ solutions/scopus/how-scopus-works/content). Scopus generates metrics on the number of documents per year, author, affiliation, journal, country, type of document, subject area and number of citations, which are crucial for scientometric analysis [31]. We used the Scopus database to identify the publication trends of OS a) in male reproductive research, b) in clinical scenarios/risk factors associated with male infertility, c) based on evaluation techniques, and d) based on prognostic/ diagnostic studies in male infertility.

\subsection{Data retrieval strategies}

The literature search was conducted on December 15, 2019 and was limited to scientific articles on humans published until 2018. Since the pioneering article on OS related to male reproduction was published in 1943 [8], the period for analysis was broken down to decades starting from 1941 (i.e. $1941-1950$, 1951-1960, 1961-1970, 1971-1980, 1981-1990, 1991-2000, 2001-2010 and 2011-2018). The TITLE-ABSKEY function was used along with multiple Boolean operators such as 'AND', 'AND NOT' and 'OR' to retrieve a maximum number of relevant articles. Furthermore, we used asterisk (*) to include all variants of the search term. The analysis was executed in four sequential steps examining the key areas of research, and keywords used for each step are listed in Supplementary Table 1.

In step 1, we retrieved scientific publications on OS related to male reproduction and analyzed the publication trend based on countries, researchers, journals, institutions and global collaborations for overall and different time periods. For subsequent steps, the scientometric data were retrieved using the search option used in step 1 along with additional keywords corresponding to the respective steps (Supplementary Table 1). In step 2, additional keywords were used to retrieve articles on OS related to specific clinical scenarios and risk factors associated with male infertility. The step 2 was divided into 10 sub-categories, namely sperm abnormalities (oligozoospermia, azoospermia, asthenozoospermia, asthenoteratozoospermia, teratozoospermia, oligoasthenozoospermia, oligoasthenoteratozoospermia and globozoospermia), varicocele, testicular cancer, erectile dysfunction, unexplained male infertility (UMI), idiopathic male infertility, male genital tract infection, obesity and metabolic syndrome, lifestyle and occupational exposure. In step 3, the articles were retrieved based on OS evaluation techniques such as direct (chemiluminescence, fluorescein isothiocyanate [FITC]-labelled lectins, electron spin resonance [ESR], dichloro-dihydro-fluorescein-diacetate [DCFH-DA], nitroblue tetrazolium [NBT], oxidation-reduction potential [ORP]) and indirect (lipid peroxidation, total antioxidant capacity [TAC], 8-hydroxy-2'-deoxyguanosine [8-OHdG], mitochondrial membrane potential [MMP], individual antioxidants) tests, using specific keywords. In step 4, publications related to prognostic/diagnostic studies on OS in male infertility were retrieved. Three independent researchers validated the title and abstract of the articles retrieved from each step and excluded non-relevant articles.

\subsection{Scientometric analysis}

The scientometric data retrieved as comma-separated value (CSV) files were converted and saved as Microsoft Excel files for descriptive statistical analysis. The geographic mapping of publication trends on OS male reproduction was carried out using Tableau Desktop (Tableau, Seattle, USA). Linear regression analysis was carried out using Microsoft Excel (2013) in order to examine the publication trend in OS. The Jonckheere-Terpstra trend test was carried out for a trend analysis in OS and different clinical scenarios using MedCalc Statistical Software (version 17.8; MedCalc Software bvba, Ostend, Belgium).

\subsection{Network and heat map analysis}

Network maps on international collaboration in OS and male reproductive research were generated for each time interval using VOSviewer software (downloaded from http://vosviewer.com) [32]. The number of documents published by each country defined the size of nodes, while the correlation between the countries was determined based on the number of co-authored documents retrieved. VOSviewer was also used to generate the heat map illustrating the top prolific authors in the area of OS and male reproductive research. 


\section{Results}

\subsection{Scientific landscape of $O S$ in male reproductive research}

The landscape analysis of scientific publications on OS related to male reproduction revealed an increasing trend from 1941 to 2018 $\left(\mathrm{R}^{2}=0.72\right)$ with a steep raise in publications starting from the time period 1991-2000 $\left(\mathrm{R}^{2}=0.81\right)$. Furthermore, the publication trend continued to increase with $31.1 \%$ and $51.6 \%$ of the total publications during the time period 2001-2010 and 2011-2018, respectively (Fig. 1).

Since 1940, the United States published the maximum number of research articles pertaining to OS in male reproduction $(n=441)$ followed by India $(n=177)$ and Italy $(n=162)$ (Fig. 2). Several countries involved in research related to OS and male reproduction had a significant number of publications $(n \geq 10)$ from the period 1991-2000, later followed an increasing trend. Among the countries, the United States topped the list with a maximum number of research publications starting from 1991 to 2000 (Table 1). The United States also collaborated with many countries from 1991 to 2000 onward. The extent of international collaboration among the different countries conducting research in this field during different time periods (1981-1990, 1991-2000, 2001-2010 and 2010-2018) are presented in Fig. 3.

The scientometric analysis revealed that scientific journals such as Fertility and Sterility $(\mathrm{n}=149)$, Andrologia $(\mathrm{n}=127)$ and Human Reproduction $(n=74)$ were the most productive in publishing articles related to OS and male reproduction (Supplementary Table 2). Publication metrics of top three journals during different time periods are provided in Supplementary Table 2.

Furthermore, scientometric data revealed Cleveland Clinic Foundation, United States $(n=198)$, University of Newcastle, Australia $(n=58)$ and All India Institute of Medical Sciences, India $(n=40)$ as the top 3 institutions contributing maximum number of publications (Fig. 4). Agarwal A ( $\mathrm{n}=197)$ was identified as the top scientist with maximum number of publications in the field of male reproduction and OS followed by Sharma R ( $\mathrm{n}=97)$ and Aitken RJ $(\mathrm{n}=83)$ during the period of 1940-2018 (Fig. 5, Supplementary Table 3). The most prolific authors in different time periods are presented in Supplementary Table 3.

\subsection{Scientific landscape of OS in clinical scenarios/risk factors associated with male infertility}

Publications on OS were analyzed considering several conditions (sperm abnormalities, varicocele, testicular cancer, erectile dysfunction, UMI, hypogonadism, male genital tract infections) and risk factors (obesity, lifestyle, occupational exposure) associated with male infertility. All the investigated conditions gained interest over the time showing an increased number of publications during different time intervals (Fig. 6). Particularly, sperm abnormalities (from 4 to 213 publications, $\mathrm{P}=0.04154$ ) and varicocele (from 0 to 171 publications, $\mathrm{P}=0.04154$ ) were the major areas investigated in relation to OS with the highest positive trend in publications from the time intervals from 1981-1990 to 2011-2018 (Fig. 6). On the other hand, publications reporting on the relationship between OS and testicular cancer showed only a small increase, from the time interval 1981-1990 to 2011-2018 $(\mathrm{P}=0.17423)$

Analysis of scientometric data based on different sperm abnormalities (azoospermia, oligozoospermia, asthenozoospermia, teratozoospermia, asthenoteratozoospermia, oligoasthenozoospermia, oligoasthenoteratozoospermia and globozoospermia) showed that the highest number of publications investigated the link between OS and asthenozoospermia (Supplementary Fig. 1A). Further analysis based on the time interval revealed an increase in the number of publications for all the investigated sperm abnormalities over different time periods except for globozoospermia (Supplementary Fig. 1B).

\subsection{Scientific landscape of OS based on specific evaluation techniques}

In the current study, scientometric analysis was conducted on the most commonly used direct and indirect tests for the evaluation of OS in male infertility. The total number of publications on indirect tests $(n=848)$ was more than thrice that of direct tests $(n=260)$ reported in the literature during the time period 1940-2018. Direct tests include chemiluminescence $(\mathrm{n}=180,69.23 \%)$, NBT $(\mathrm{n}=26,10 \%)$, ESR ( $\mathrm{n}=18,6.92 \%)$, ORP $(\mathrm{n}=14,5.38 \%)$, DCFH-DA $(\mathrm{n}=12,4.62 \%)$ and FITC-labelled lectins ( $\mathrm{n}=10,3.85 \%)$, while indirect tests include individual antioxidant assays $(\mathrm{n}=300,35.38 \%)$, lipid peroxidation $(\mathrm{n}=252,29.72 \%)$, TAC $(\mathrm{n}=130,15.33 \%)$, MMP $(\mathrm{n}=97,11.44 \%)$ and 8-OHdG ( $\mathrm{n}=69,8.14 \%$ ) (Fig. 7).

Our results revealed that the chemiluminescence technique was the only direct test that was used during the 1981-1990 time period. Though new techniques such as ESR $(n=10)$, NBT $(n=4)$ and FITClabelled lectins $(\mathrm{n}=1)$ surfaced during 1991-2000, and DCFH-DA $(n=5)$ during 2001-2010, yet chemiluminescence based publications were predominant in those periods as well ( $n=61$ and $n=64$, respectively) (Fig. 7A). Even though chemiluminescence dominated the period 2011-2018, it was interesting to note that ORP had 14 publications in a short span of 2 years (2017-2018).

All indirect tests showed an increasing trend in publication over the time period (Fig. 7B). Lipid peroxidation assay was the first indirect test introduced to evaluate the OS in semen samples (during the time period 1971-1980). However, since 1981, the number of publications based on individual antioxidants $(n=300)$ were more compared to lipid peroxidation $(\mathrm{n}=251)$ (Fig. $7 \mathrm{~B})$. The other three indirect tests-based publications (MMP, TAC, and 8-OHdG) were observed from the time period 1991-2000. During the time period 2011-2018, both the individual antioxidant assay and lipid peroxidation assay had equal number of publications $(\mathrm{n}=133$ ) (Fig. 7B).

\subsection{Landscape analysis of prognostic/diagnostic studies on OS in male infertility}

Based on the scientometric data, the number of publications on the prognostic/diagnostic value of OS evaluation increased strongly over the time, reaching a maximum $(n=155)$ in the last time interval (2011-2018) (Fig. 8). Out of 2108 studies published on OS in male reproductive research, prognostic and diagnostic based studies represent $13.6 \%$ of the total number of publications, with Fertility and Sterility ( $\mathrm{n}=18 ; 6.3 \%)$, Andrologia $(\mathrm{n}=17 ; 5.9 \%)$ and Human Reproduction ( $\mathrm{n}=13 ; 4.5 \%$ ) being the most fruitful journals. We also identified Agarwal, A ( $\mathrm{n}=52 ; 18.1 \%)$, Sharma, $\mathrm{R}(\mathrm{n}=23 ; 8 \%)$ and

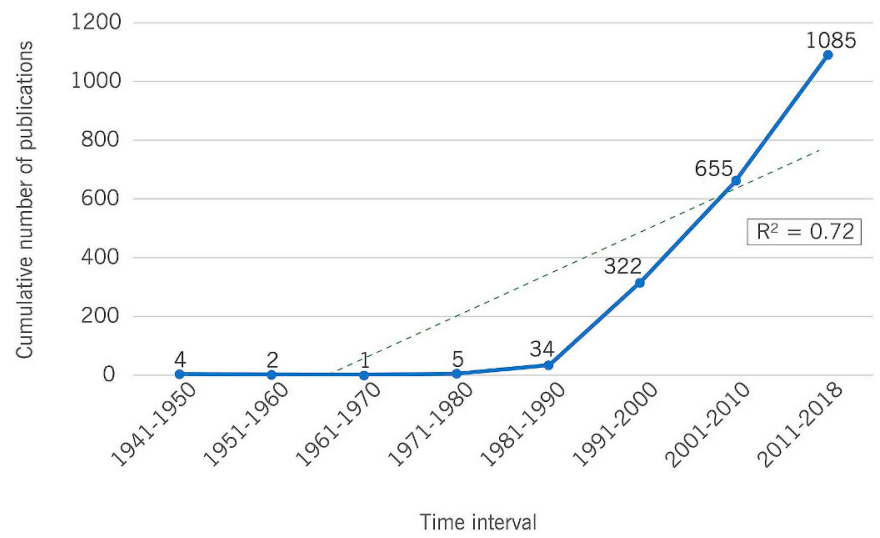

Fig. 1. Cumulative publications on oxidative stress in male reproductive research during different time periods. 


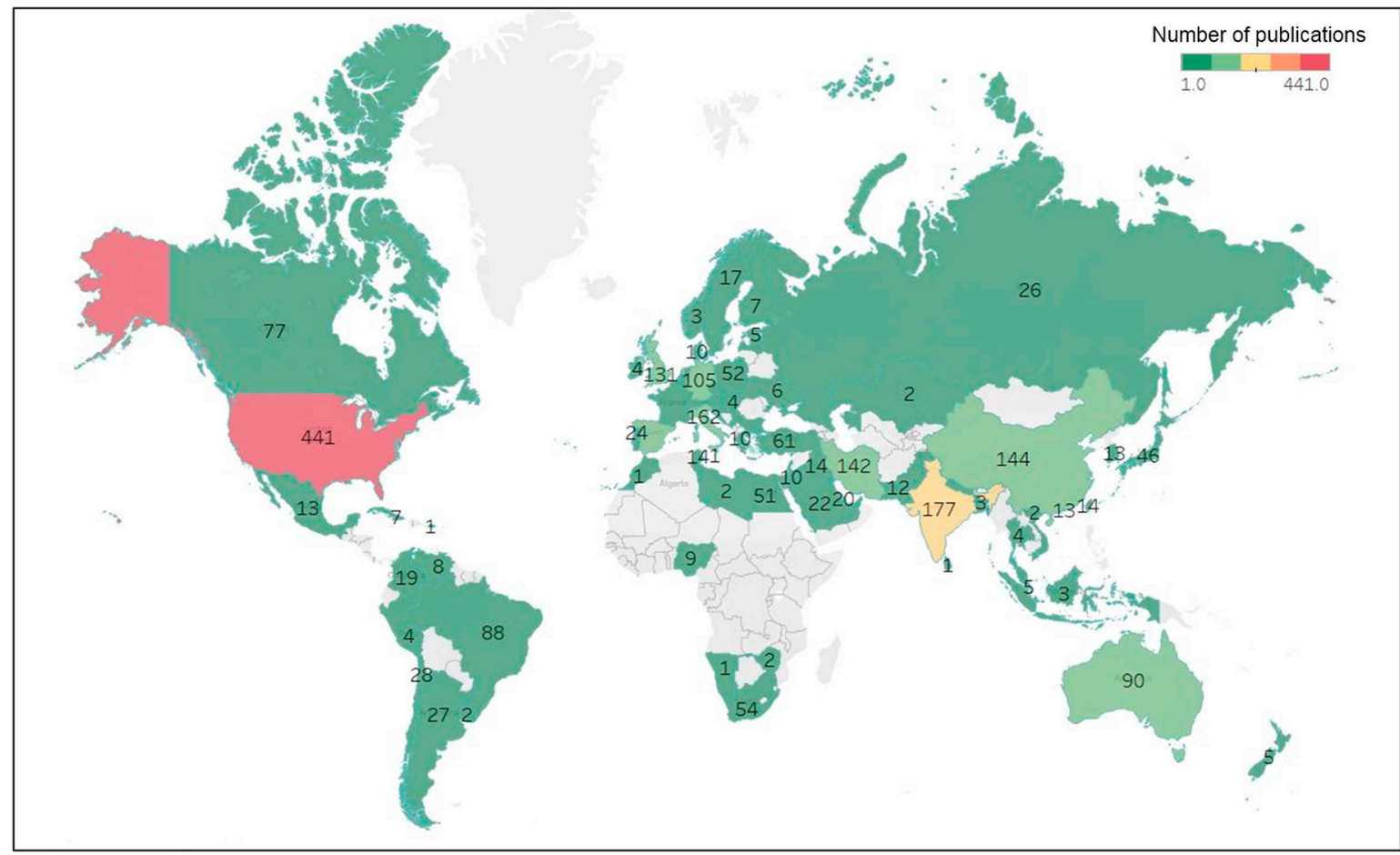

Fig. 2. Analysis of research trends based on the origin of publications across the globe.

Table 1

Top 10 countries based on the total number of publications during different time periods.

\begin{tabular}{|c|c|c|c|c|c|c|c|c|c|c|c|}
\hline Total & & 1971-1980 & & 1981-1990 & & 1991-2000 & & $2001-2010$ & & $2011-2018$ & \\
\hline Country & $\mathrm{N}$ of docs & Country & $\mathrm{N}$ of docs & Country & $\mathrm{N}$ of docs & Country & $\mathrm{N}$ of docs & Country & $\mathrm{N}$ of docs & Country & $\mathrm{N}$ of docs \\
\hline United States & $\begin{array}{l}441 \\
(20.9 \%)\end{array}$ & United States & $\begin{array}{l}2 \\
(40 \%)\end{array}$ & India & $\begin{array}{l}6 \\
(17.6 \%)\end{array}$ & United States & $\begin{array}{l}75 \\
(23.3 \%)\end{array}$ & United States & $\begin{array}{l}144 \\
(22.0 \%)\end{array}$ & United States & $\begin{array}{l}219 \\
(20.2 \%)\end{array}$ \\
\hline India & $\begin{array}{l}177 \\
(8.4 \%)\end{array}$ & Belgium & $\begin{array}{l}1 \\
(20 \%)\end{array}$ & United Kingdom & $\begin{array}{l}5 \\
(14.7 \%)\end{array}$ & United Kingdom & $\begin{array}{l}60 \\
(18.6 \%)\end{array}$ & India & $\begin{array}{l}52 \\
(7.9 \%)\end{array}$ & Iran & $\begin{array}{l}116 \\
(10.7 \%)\end{array}$ \\
\hline Italy & $\begin{array}{l}162 \\
(7.7 \%)\end{array}$ & Germany & $\begin{array}{l}1 \\
(20 \%)\end{array}$ & France & $\begin{array}{l}4 \\
(11.8 \%)\end{array}$ & Germany & $\begin{array}{l}22 \\
(6.8 \%)\end{array}$ & Italy & $\begin{array}{l}48 \\
(7.3 \%)\end{array}$ & India & $\begin{array}{l}101 \\
(9.3 \%)\end{array}$ \\
\hline China & $\begin{array}{l}144 \\
(6.8 \%)\end{array}$ & Undefined & $\begin{array}{l}1 \\
(20 \%)\end{array}$ & Sweden & $\begin{array}{l}3 \\
(8.8 \%)\end{array}$ & Canada & $\begin{array}{l}21 \\
(6.5 \%)\end{array}$ & China & $\begin{array}{l}46 \\
(7.0 \%)\end{array}$ & Italy & $\begin{array}{l}91 \\
(8.4 \%)\end{array}$ \\
\hline Iran & $\begin{array}{l}142 \\
(6.7 \%)\end{array}$ & & & Germany & $\begin{array}{l}3 \\
(8.8 \%)\end{array}$ & Italy & $\begin{array}{l}21 \\
(6.6 \%)\end{array}$ & United Kingdom & $\begin{array}{l}40 \\
(6.1 \%)\end{array}$ & China & $\begin{array}{l}89 \\
(8.2 \%)\end{array}$ \\
\hline United Kingdom & $\begin{array}{l}131 \\
(6.2 \%)\end{array}$ & & & Argentina & $\begin{array}{l}2 \\
(5.9 \%)\end{array}$ & India & $\begin{array}{l}18 \\
(5.6 \%)\end{array}$ & Australia & $\begin{array}{l}36 \\
(5.5 \%)\end{array}$ & Brazil & $\begin{array}{l}74 \\
(6.8 \%)\end{array}$ \\
\hline Germany & $\begin{array}{l}106 \\
(5.0 \%)\end{array}$ & & & Australia & $\begin{array}{l}2 \\
(5.9 \%)\end{array}$ & France & $\begin{array}{l}14 \\
(4.3 \%)\end{array}$ & Spain & $\begin{array}{l}36 \\
(5.5 \%)\end{array}$ & Germany & $\begin{array}{l}47 \\
(4.3 \%)\end{array}$ \\
\hline Australia & $\begin{array}{l}90 \\
(4.3 \%)\end{array}$ & & & Italy & $\begin{array}{l}2 \\
(5.9 \%)\end{array}$ & Japan & $\begin{array}{l}14 \\
(4.3 \%)\end{array}$ & Germany & $\begin{array}{l}33 \\
(5.0 \%)\end{array}$ & Spain & $\begin{array}{l}47 \\
(4.3 \%)\end{array}$ \\
\hline Spain & $\begin{array}{l}89 \\
(4.2 \%)\end{array}$ & & & United States & $\begin{array}{l}1 \\
(2.9 \%)\end{array}$ & Belgium & $\begin{array}{l}12 \\
(3.7 \%)\end{array}$ & France & $\begin{array}{l}32 \\
(4.9 \%)\end{array}$ & Australia & $\begin{array}{l}45 \\
(4.1 \%)\end{array}$ \\
\hline Brazil & $\begin{array}{l}88 \\
(4.2 \%)\end{array}$ & & & Japan & $\begin{array}{l}1 \\
(2.9 \%)\end{array}$ & Croatia & $\begin{array}{l}10 \\
(3.1 \%)\end{array}$ & Iran & $\begin{array}{l}26 \\
(4.0 \%)\end{array}$ & South Africa & $\begin{array}{l}37 \\
(3.4 \%)\end{array}$ \\
\hline
\end{tabular}

Aitken, RJ ( $\mathrm{n}=14 ; 4.9 \%)$ as the top three scientists in prognostic/ diagnostic studies on OS.

\section{Discussion}

Over the past several decades, the relationship between ROS and sperm functions has been well established in the literature [33,34]. ROS play a dual role in male reproduction, supporting and triggering physiological functions of spermatozoa within critical levels while having significant adverse consequences on male fertility at high concentrations [21]. In this study, we conducted a landscape analysis of the literature on OS in male reproductive research to explore the trends in publication during various time periods. Our analysis revealed an increased number of publications from 1941 to 2018 (over 1000-fold increase) which signifies the growing importance of ROS and OS in the field of male reproductive research. However, a period-wise analysis revealed fewer publications until the 1981 to 1990 time frame. A plethora of evidence on the physiological role of ROS in sperm capacitation, hyperactivation, acrosome reaction, as well as sperm-oocyte fusion were published from the 1990s onwards [35-37]. These findings could have further inculcated an interest in researchers to conduct more research in order to understand the cellular mechanisms, which was evident with the steep raise in publications as well as an increase in the collaboration between the researchers across countries during the 1991-2000 period. In addition, statistical data published in 1997 reported that OS is one of the major causes of male infertility in the 

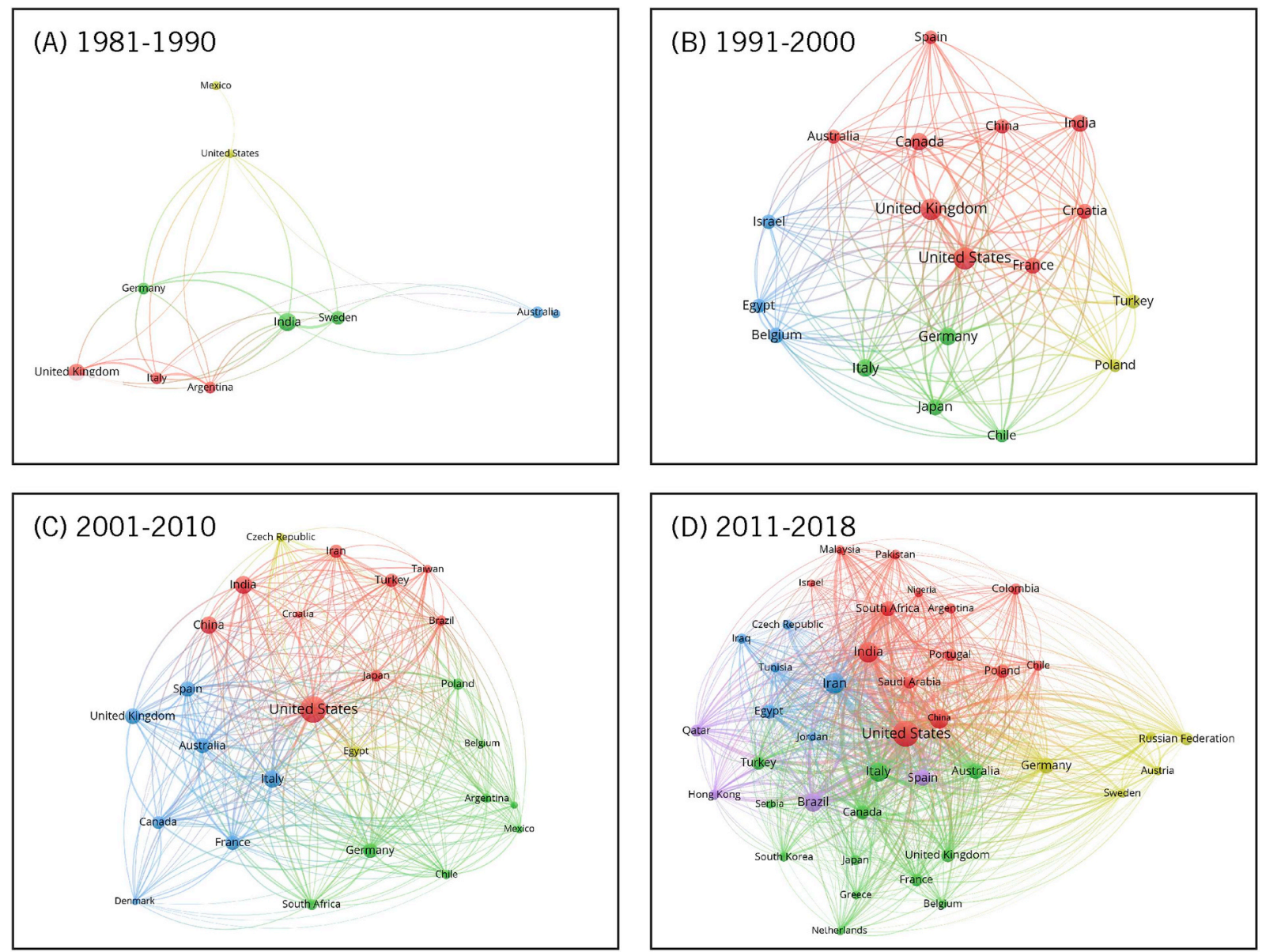

Fig. 3. International collaborations during different time periods (A) 1981-1990, (B) 1991-2000, (C) 2001-2010 and (D) 2011-2018.

United States accounting for $30 \%-40 \%$ of infertile men [38]. This could have further triggered an extensive research and explains the sudden rise in the number of publications from the United States during the time period 2001-2010, which contributed towards one-fifth of the total number of publications $(22 \%)$. Furthermore, the current study results revealed Cleveland Clinic, USA, as the leading institute in OSrelated male reproductive research, which corroborates with our previous scientometric findings [29].

The relationship between clinical scenarios and OS has been widely investigated since 1991 and semen abnormalities were found to be the most investigated scenario in association with OS. In fact, semen parameters such as sperm concentration, motility, and morphology are reportedly affected by the oxidation of cellular components and induction of apoptotic pathway [2,18]. Our scientometric analysis

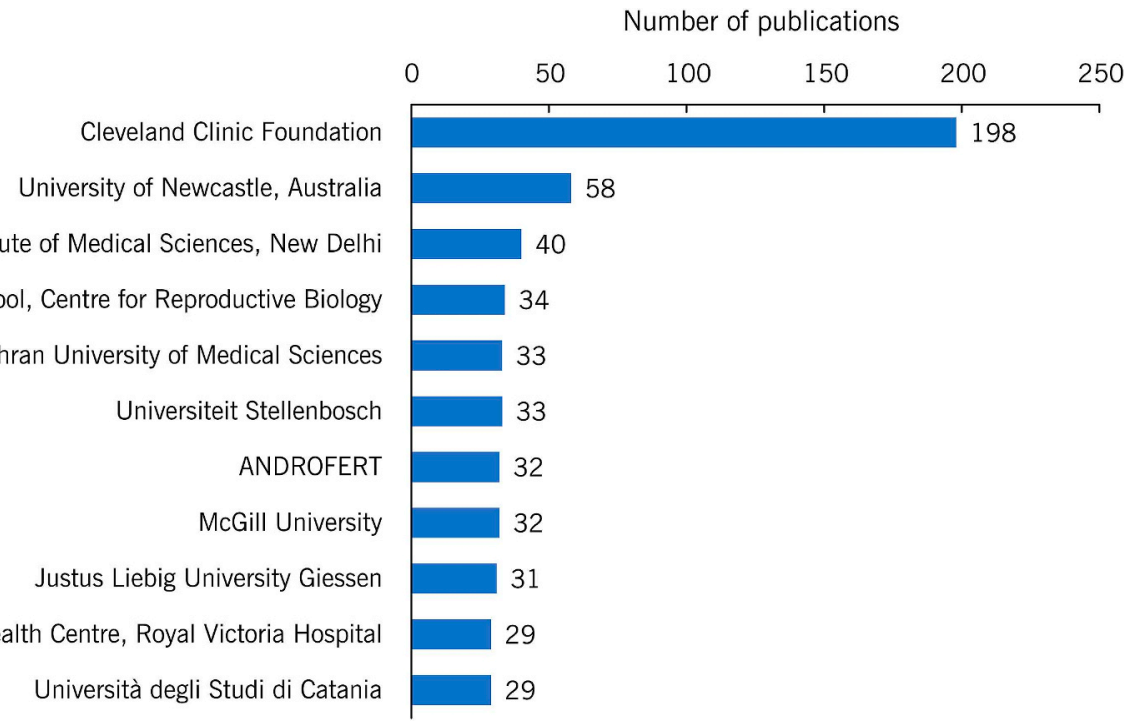

Fig. 4. Top 10 institutions based on number of publications on oxidative stress in male reproductive research. 


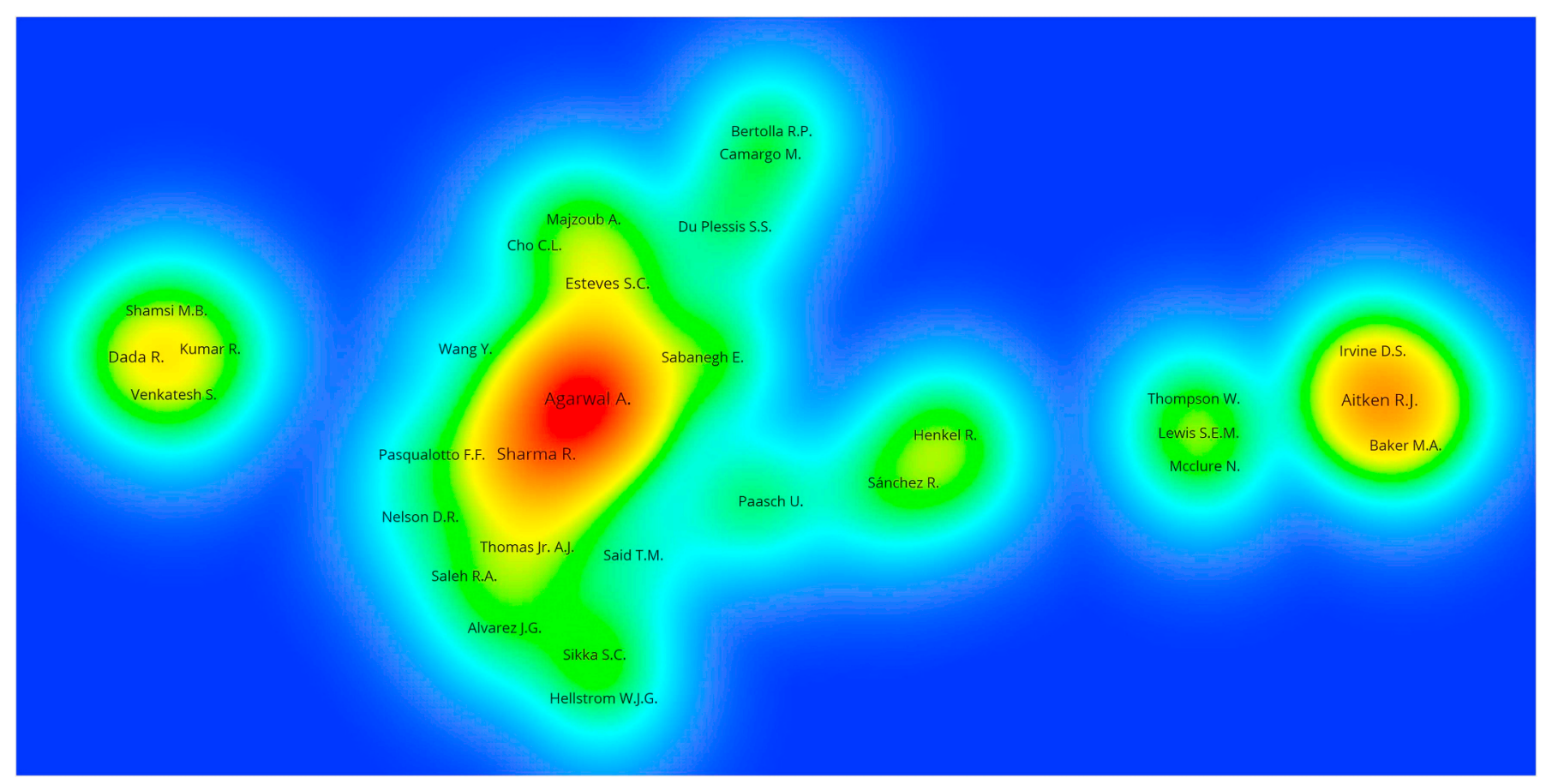

Fig. 5. Heat map illustrating the most prolific authors.

revealed the highest number of publications on asthenozoospermia and OS indicating the importance of this relationship in the pathogenesis of male infertility. In fact, sperm motility has been demonstrated to be the first parameter affected by OS [39]. The generation of sperm motility is a complex process that depends on mitochondrial ATP as source of energy [40]. OS reportedly disturbs the MMP and the associated electron transport chain, impairing sperm motility [41]. Therefore, the evaluation of OS could be an important assessment in the identification of etiology of sperm abnormalities, particularly in asthenozoospermic patients.

Varicocele was found to be the second most investigated clinical scenario in association with OS. It is a vascular disease where the pampiniform venous plexus and the internal spermatic veins significantly enlarge [42]. The prevalence of varicocele in the global population is $15 \%-20 \%$, while in infertile population it rises to $25 \%-40 \%$ [42]. Therefore, elucidation of mechanisms involved in varicocelemediated infertility is of great interest. In varicocele, spermatogenesis is affected due to multiple factors such as hypoxia, hyperthermia, metabolite reflux and epididymis dysfunction as well as the establishment of an oxidative microenvironment [43]. Infertile men with varicocele reportedly showed higher ROS levels as well as reduced antioxidant concentrations [44] and the grade of varicocele is associated with the degree of OS [45]. Moreover, the effect of varicocelectomy on oxidative markers has been investigated, showing post-operatively reduced levels

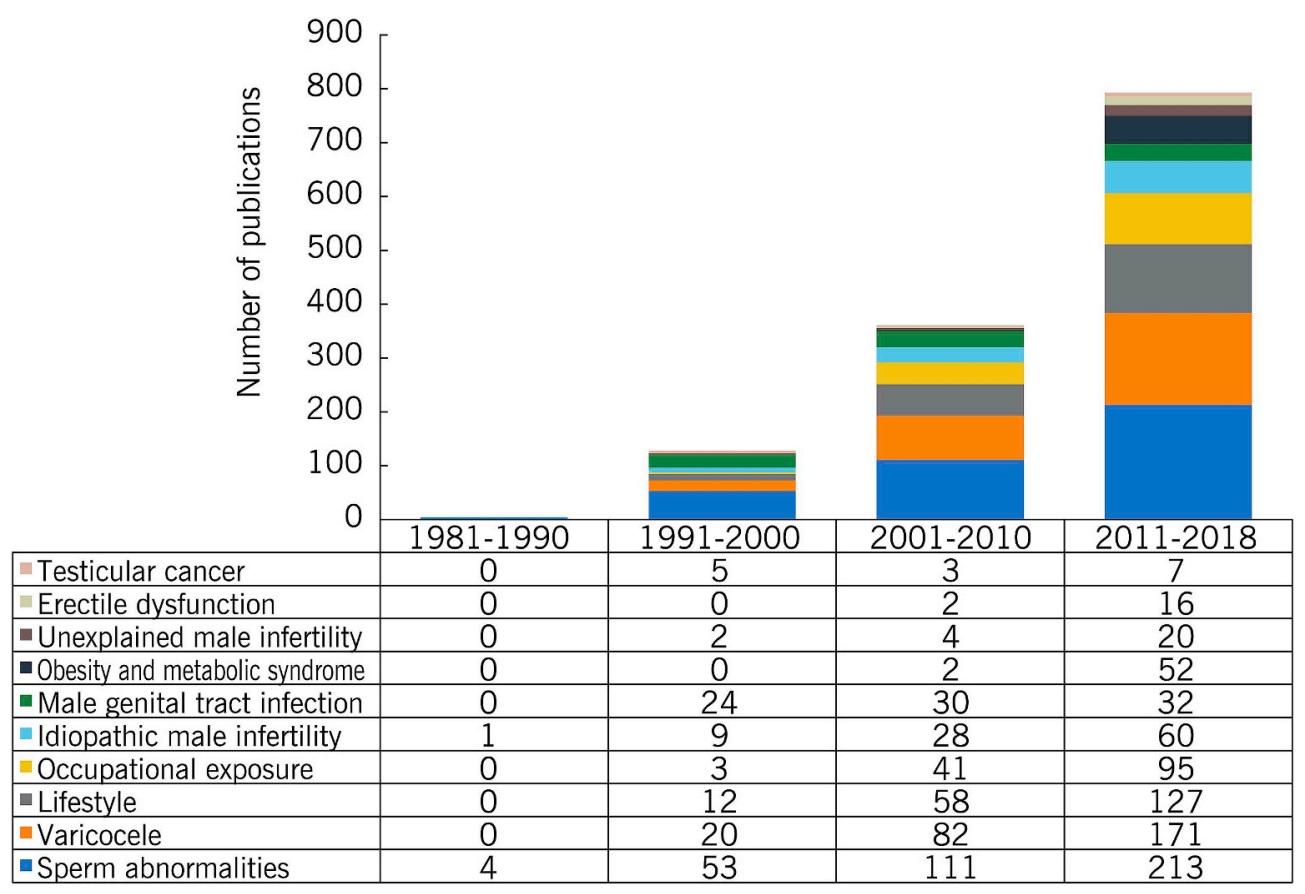

Fig. 6. Analysis of publications during different time periods based on clinical scenarios/risk factors associated with male infertility. 
(A)

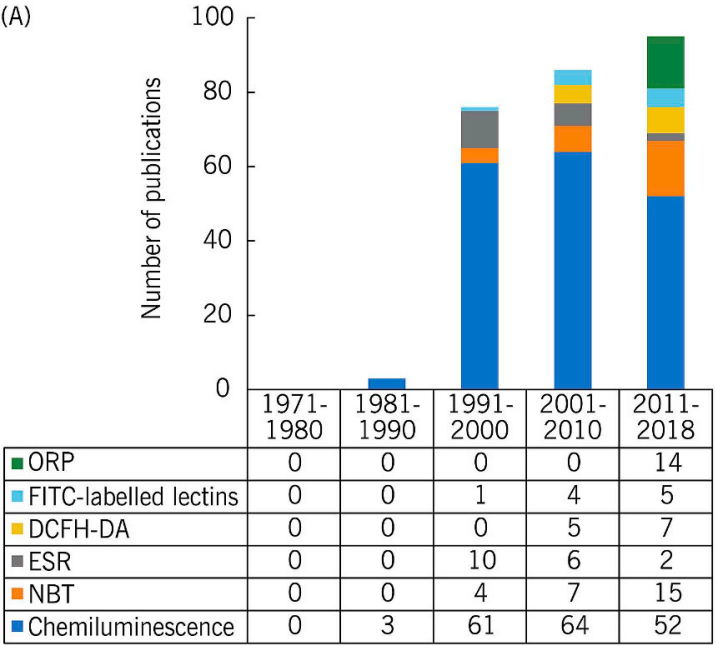

(B)

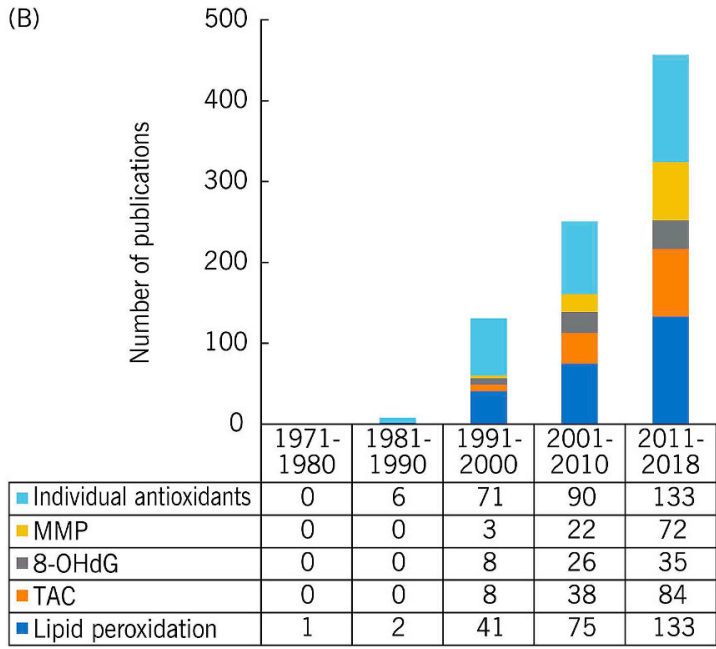

Fig. 7. Analysis of publications during different time periods based on specific oxidative stress evaluation techniques. ORP, oxidation-reduction potential; FITC, fluorescein isothiocyanate; DCFH-DA, dichloro-dihydro-fluorescein-diacetate; ESR, electron spin resonance; NBT, nitroblue tetrazolium; MMP, mitochondrial membrane potential; 8-OHdG, 8-hydroxy-2'-deoxyguanosine; TAC, total antioxidant capacity.

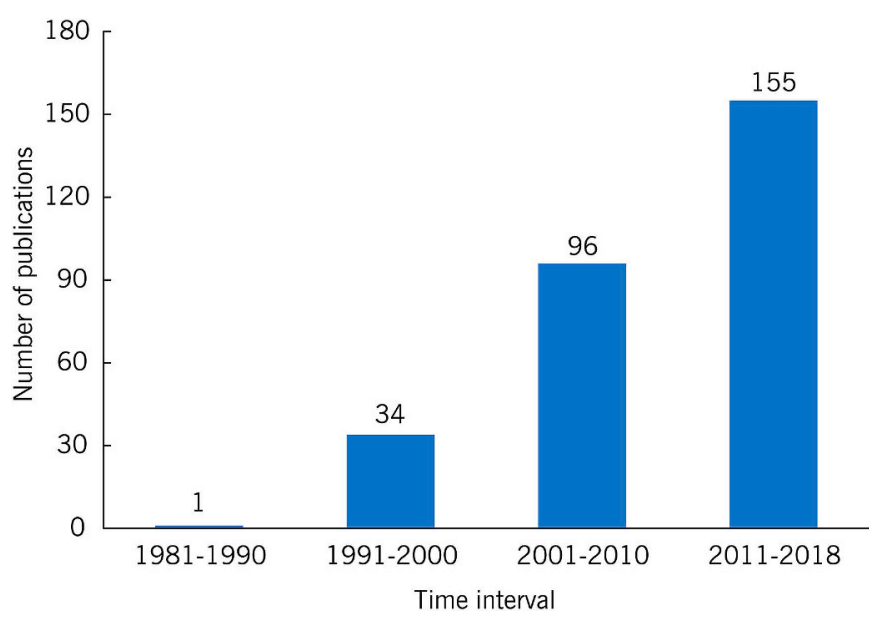

Fig. 8. Cumulative publications on prognostic/diagnostic studies on oxidative stress during different time periods.

of ROS concentration [46]. Since varicocelectomy has been associated with increased clinical pregnancy and live birth rates [47], the investigation of $O S$ as a causative factor of testicular damage may provide a prognostic factor for the fertility status after varicocelectomy.

Testicular cancer and globozoospermia were the least investigated scenarios in association with OS. This was expected, as a genetic etiology has been identified for both $[48,49]$. The incidence of testicular cancer has increased over the years among men aged 15-35 years [50] and several studies have investigated the genetic and epigenetic alterations as pathogenic factors, with many differences between seminoma and non-seminoma tumors [51,52]. The investigation of seminal features such as OS may also be limited by the necessity to treat cancer promptly as patients can utilize cryopreservation and assisted reproductive techniques (ART) to achieve a pregnancy once the treatment is over. Similar considerations may be valid for globozoospermia, a genetic condition characterized by the absence of a complete acrosome [53]. The low incidence in the general population $(<0.1 \%)$ as well as the possibility to overcome the fertility issues with intracytoplasmic sperm injection (ICSI) could make such conditions less investigated [53].

Over the years, extensive research has been carried out to establish a link between seminal OS and male infertility, which lead to the advent of more than 30 different assays for measuring seminal OS. Direct assays measure only ROS whereas indirect assays assess the levels of antioxidants or evaluate the detrimental effects of OS on sperm cell membrane, mitochondria or DNA [54]. The increased number of publications on indirect tests compared to direct tests signifies that the researchers are keen on studying the consequences of OS rather than the actual measurement of ROS. Among the direct tests, chemiluminescence was the most widely used technique to measure ROS, which could be correlated to high intra- and inter-observer reliability as well as high reproducibility reported for this assay [55]. Furthermore, the validation of the chemiluminescence assay in a clinical setup demonstrated it to be a highly reliable and accurate diagnostic test [54]. This could have favored this assay for the evaluation of seminal OS by several laboratories globally, thus reflecting an increase in the number of publications based on chemiluminescence during the time period 2001-2010. Later, physiological and pathological levels of ROS in infertile men were published by Cleveland clinic [56,57]. All the above justifies the widespread use of the chemiluminescence technique.

A novel direct assay involving the measurement of ORP in semen was published in 2016 [47]. Unlike other cumbersome techniques, this quick and easy-to-use assay provides a global picture on the balance between oxidants and antioxidants [58] rendering a considerable number of publications ( $\mathrm{n}=11,4.23 \%$ ) in a short span of two years which is in par with the publications based on the most widely used chemiluminescence technique $(n=11,4.23 \%)$ during the same time period. This suggests that ORP might be the future direct assay to measure OS in semen samples. The application of ORP measurement in clinical practice is expected to benefit the management of infertile men with pathology as well as to predict the success of assisted reproductive techniques $[47,59]$. Furthermore, a multi-center study reported an ORP cut-off value of $1.34 \mathrm{mV} / 10^{6}$ sperm $/ \mathrm{mL}$ to distinguish fertile from infertile men [60].

Among the indirect assays, the measurement of different antioxidants was reported in maximum number of publications. Unlike other indirect or direct tests that measured only one parameter, the articles retrieved under individual antioxidants group measured one or more of the seven most important antioxidants present in semen, namely superoxide dismutase, catalase, glutathione peroxidase, ascorbic acid, glutathione, thioredoxin, and glutathione reductase. The studies relating to the mechanism and effects of each antioxidant explain the increased number of publications under this category. The standardization of the TAC assay and the establishment of diagnostic cut-offs could have increased its use resulting in the growing number of publications on the TAC assay $[59,61]$. Publications related to assays 
such as MMP, 8-OHdG and lipid peroxidation that measure the effect of OS on spermatozoa increased steadily over the time periods with lipid peroxidation having the highest number of publications. However, in the last time period (2011-2018), evaluation of antioxidants and lipid peroxidation gained equal importance. Since lipid peroxidation can compromise the structural and functional integrity of spermatozoa, increased attention is being paid to this parameter.

Standard semen analysis is used to analyze the male fertility potential. However, it fails to characterize infertile patients with normal semen parameters who are classified as unexplained male infertility [62]. Similarly, it cannot identify an etiological cause for idiopathic infertility where the reason for altered semen parameters is unknown [63]. Hence, there is a necessity of new molecular markers as prognostic and diagnostic tools in male infertility assessment. In this context, we analyzed the publication trends on the prognostic and diagnostic studies on OS in the evaluation of male infertility. A prognostic study evaluates the influence of specific scenarios and risk factors on the outcome of a disease [64], while a diagnostic study aims to identify variables for the determination of a pathological condition [65]. The results of this study clearly show a significant increase in the scientific interest in the investigation of prognostic/diagnostic value of OS evaluation. An increased number of publications on OS was observed in a previous scientometric study where its relatedness with SDF was reported [29]. It has been shown that about $70 \%$ of men with idiopathic infertility have elevated levels of seminal ROS [66]. Furthermore, the major role of OS in the pathogenesis of several male infertility-related scenarios such as varicocele [67], chronic prostatitis [68], asthenozoospermia [69] and idiopathic infertility [70] has been well documented. In fact, several studies have emphasized the emergence of seminal OS status as a significant prognostic and diagnostic tool in the evaluation of male infertility $[59,71,72]$. Furthermore, highlighting the importance of OS in male infertility, a recent collaborative article by 91 authors from six continents and 26 countries proposed a novel clinical practice guideline for the management of OS mediated idiopathic male infertility. The authors recommended a new paradigm for the diagnosis and management of infertile men with OS [20]. These considerations significantly highlight the role of OS in prognosis and diagnosis of male infertility, explaining the increased number of publications in this field over the time.

\section{Conclusion}

Our comprehensive scientometric analysis revealed an increasing trend in OS-based male reproductive research starting from the time period 1991-2000. Semen abnormalities, particularly, asthenozoospermia, and varicocele were the highly investigated clinical scenarios in association with OS. Chemiluminescence was the most widely used direct technique to assess OS while measurement of antioxidants and lipid peroxidation were the most commonly used indirect tests. Our analysis emphasizes the emergence of OS as a key prognostic and diagnostic parameter in the evaluation of male infertility.

\section{Future perspectives}

OS is directly associated with several male infertility scenarios, and its diagnosis and management are crucial. The increased number of publications on OS, particularly, on the prognostic/diagnostic value of OS evaluation, emphasizes OS as the primary mediator of male infertility and thus, mitigating its effects as one of the main management options. Several clinical trials have been conducted to investigate the efficacy of antioxidant therapy in the management of OS mediated male infertility [73-76]. Furthermore, OS and the associated increase in sperm DNA damage has been well-established to affect ART outcomes $[77,78]$. High sperm DNA integrity is directly associated with improved fertilization rate and embryonic development in in vitro fertilization (IVF) and ICSI $[79,80]$. Therefore, idiopathic infertile patients undergoing ART procedure will benefit by evaluating OS. In future, inclusion of OS testing in routine male fertility evaluation would greatly facilitate early diagnosis and management of OS-mediated male infertility, particularly, idiopathic infertility, and serve as a better predictor of ART outcomes.

\section{CRediT authorship contribution statement}

Ashok Agarwal: Conceptualization, Writing - review \& editing. Saradha Baskaran: Data curation, Writing - original draft, Writing review \& editing. Manesh Kumar Panner Selvam: Formal analysis, Writing - original draft. Renata Finelli: Writing - original draft. Catalina Barbarosie: Writing - original draft. Kathy Amy Robert: Formal analysis, Writing - original draft. Concetta Iovine: Formal analysis, Writing - original draft. Kruyanshi Master: Formal analysis, Writing - original draft. Ralf Henkel: Formal analysis, Writing - original draft.

\section{Declaration of competing interest}

The authors declare no conflict of interest.

\section{Acknowledgement}

The authors are grateful to the following for their critical review of our manuscript and helpful suggestions: Drs. Mohamed Arafa (Hamad Medical Corporation, Qatar), Dr. Peter Natesan Pushparaj (King Abdulaziz University, Jeddah, Kingdom of Saudi Arabia), Dr. Kristian Leisegang (University of the Western Cape, South Africa), Dr. Neel Parekh (Cleveland Clinic, USA), Dr. Pallav Sengupta (MAHSA University, Jenjarom, Selangor, Malaysia), Dr. Edmund Ko (Loma Linda University Health, Loma Linda), Drs. Rakesh Sharma and Rafael Ambar (American Center for Reproductive Medicine, Cleveland Clinic). The authors thank Ken Abraham and Susan Kido from the Center for Medical Art and Photography for assistance with the figures. Authors also wish to thank the American Center for Reproductive Medicine for support of this research.

\section{Appendix A. Supplementary data}

Supplementary data to this article can be found online at https:// doi.org/10.1016/j.freeradbiomed.2020.05.008.

\section{Funding}

Research support for this study was provided by the American Center for Reproductive Medicine, Cleveland Clinic.

\section{References}

[1] A.J. Kattoor, et al., Oxidative stress in atherosclerosis, Curr. Atherosclerosis Rep. 19 (11) (2017) 42

[2] S.K. Bardaweel, et al., Reactive oxygen species: the dual role in physiological and pathological conditions of the human body, Eurasian J. Med. 50 (3) (2018) 193-201.

[3] A. Agarwal, K. Makker, R. Sharma, Clinical relevance of oxidative stress in male factor infertility: an update, Am. J. Reprod. Immunol. 59 (1) (2008) 2-11.

[4] I. Liguori, et al., Oxidative stress, aging, and diseases, Clin. Interv. Aging 13 (2018) 757.

[5] A. Agarwal, et al., Role of oxidative stress, infection and inflammation in male infertility, Andrologia 50 (11) (2018) e13126.

[6] Y.Z. Fang, S. Yang, G. Wu, Free radicals, antioxidants, and nutrition, Nutrition 18 (10) (2002) 872-879.

[7] J. MacLeod, The role of oxygen in the metabolism and motility of human spermatozoa, Am. J. Physiol. Legacy Content 138 (3) (1943) 512-518.

[8] J. Tosic, A. Walton, Formation of hydrogen peroxide by spermatozoa and its inhibitory effect of respiration, Nature 158 (1946) 485.

[9] R. Jones, T. Mann, R. Sherins, Peroxidative breakdown of phospholipids in human spermatozoa, spermicidal properties of fatty acid peroxides, and protective action of seminal plasma, Fertil. Steril. 31 (5) (1979) 531-537. 
[10] R. Jones, T. Mann, Lipid peroxidation in spermatozoa, Proc. R. Soc. Lond. B Biol. Sci. 184 (1074) (1973) 103-107.

[11] R. Jones, T. Mann, Toxicity of exogenous fatty acid peroxides towards spermatozoa, J. Reprod. Fertil. 50 (2) (1977) 255-260.

[12] R. Jones, T. Mann, R.J. Sherins, Adverse effects of peroxidized lipid on human spermatozoa, Proc. R. Soc. Lond. B Biol. Sci. 201 (1145) (1978) 413-417.

[13] R.J. Aitken, J.S. Clarkson, Cellular basis of defective sperm function and its association with the genesis of reactive oxygen species by human spermatozoa, J. Reprod. Fertil. 81 (2) (1987) 459-469.

[14] R.J. Aitken, et al., Redox regulation of tyrosine phosphorylation in human spermatozoa and its role in the control of human sperm function, J. Cell Sci. 108 (Pt 5) (1995) 2017-2025.

[15] E. de Lamirande, C. Gagnon, Human sperm hyperactivation and capacitation as parts of an oxidative process, Free Radic. Biol. Med. 14 (2) (1993) 157-166.

[16] J. Rivlin, et al., Role of hydrogen peroxide in sperm capacitation and acrosome reaction, Biol. Reprod. 70 (2) (2004) 518-522.

[17] S.S. Du Plessis, et al., Contemporary evidence on the physiological role of reactive oxygen species in human sperm function, J. Assist. Reprod. Genet. 32 (4) (2015) 509-520.

[18] E. Barati, H. Nikzad, M. Karimian, Oxidative stress and male infertility: current knowledge of pathophysiology and role of antioxidant therapy in disease management, Cell. Mol. Life Sci. 77 (1) (2020) 93-113.

[19] F.M. Lanzafame, et al., Oxidative stress and medical antioxidant treatment in male infertility, Reprod. Biomed. Online 19 (5) (2009) 638-659.

[20] A. Agarwal, et al., Male oxidative stress infertility (MOSI): proposed terminology and clinical practice guidelines for management of idiopathic male infertility, World J. Mens. Health 37 (3) (2019) 296-312.

[21] A.T. Alahmar, Role of oxidative stress in male infertility: an updated review, J. Hum. Reprod. Sci. 12 (1) (2019) 4-18.

[22] R.J. Aitken, et al., Oxidative stress and male reproductive health, Asian J. Androl. 16 (1) (2014) 31-38.

[23] S. Bisht, et al., Oxidative stress and male infertility, Nat. Rev. Urol. 14 (8) (2017) $470-485$.

[24] A. Agarwal, S. Prabakaran, S.S. Allamaneni, Relationship between oxidative stress, varicocele and infertility: a meta-analysis, Reprod. Biomed. Online 12 (5) (2006) 630-633.

[25] G.G. Karuga, P.B. Lowry, V.J. Richardson, Assessing the impact of premier information systems research over time, Commun. AIS (CAIS) 19 (2007) 115-131.

[26] G. Gonzalez-Alcaide, et al., Coauthorship networks and institutional collaboration patterns in reproductive biology, Fertil. Steril. 90 (4) (2008) 941-956.

[27] R. Aleixandre-Benavent, C. Simon, B.C. Fauser, Trends in clinical reproductive medicine research: 10 years of growth, Fertil. Steril. 104 (1) (2015) 131-7.e5.

[28] Y. Zhang, et al., Research trends and perspectives of male infertility: a bibliometric analysis of 20 years of scientific literature, Andrology 4 (6) (2016) 990-1001.

[29] S. Baskaran, et al., An In-Depth Bibliometric Analysis and Current Perspective on Male Infertility Research, World J Mens Health, 2019.

[30] A. Agarwal, et al., Unraveling the footsteps of proteomics in male reproductive research: a scientometric approach, Antioxidants Redox Signal. 32 (8) (2020) 536-549.

[31] A. Agarwal, et al., Bibliometrics: tracking research impact by selecting the appropriate metrics, Asian J. Androl. 18 (2) (2016) 296-309.

[32] N.J. Van Eck, L. Waltman, Text Mining and Visualization Using VOSviewer, (2011) 1109.2058 .

[33] K. Tremellen, Oxidative stress and male infertility-a clinical perspective, Hum. Reprod. Update 14 (3) (2008) 243-258.

[34] R.J. Aitken, Reactive oxygen species as mediators of sperm capacitation and pathological damage, Mol. Reprod. Dev. 84 (10) (2017) 1039-1052.

[35] E. De Lamirande, C. Gagnon, Impact of reactive oxygen species on spermatozoa: a balancing act between beneficial and detrimental effects, Hum. Reprod. 10 (suppl_1) (1995) 15-21.

[36] C. Lector, Oxidative stress and role of antioxidants in normal and abnormal sperm function, Front. Biosci. 1 (1996) e78-86.

[37] J. Griveau, D.L. Lannou, Reactive oxygen species and human spermatozoa: physiology and pathology, Int. J. Androl. 20 (2) (1997) 61-69.

[38] F.M. Lanzafame, et al., Oxidative stress and medical antioxidant treatment in male infertility, Reprod. Biomed. Online 19 (5) (2009) 638-659.

[39] M. Dobrakowski, et al., Oxidative stress and motility impairment in the semen of fertile males, Andrologia 49 (10) (2017).

[40] M. Plaza Davila, et al., Inhibition of mitochondrial complex I leads to decreased motility and membrane integrity related to increased hydrogen peroxide and reduced ATP production, while the inhibition of glycolysis has less impact on sperm motility, PLoS One 10 (9) (2015) e0138777.

[41] P. Uribe, et al., Peroxynitrite-mediated nitrosative stress decreases motility and mitochondrial membrane potential in human spermatozoa, Mol. Hum. Reprod. 21 (3) (2015) 237-243.

[42] C.K. Naughton, A.K. Nangia, A. Agarwal, Pathophysiology of varicoceles in male infertility, Hum. Reprod. Update 7 (5) (2001) 473-481.

[43] B. Alsaikhan, et al., Epidemiology of varicocele, Asian J. Androl. 18 (2) (2016) 179-181.

[44] A. Hamada, S.C. Esteves, A. Agarwal, Insight into oxidative stress in varicoceleassociated male infertility: part 2, Nat. Rev. Urol. 10 (1) (2013) 26-37.

[45] I.T. Koksal, et al., The role of reactive oxygen species in testicular dysfunction associated with varicocele, BJU Int. 86 (4) (2000) 549-552.

[46] S.A. Abdelbaki, et al., The impact of coexisting sperm DNA fragmentation and seminal oxidative stress on the outcome of varicocelectomy in infertile patients: a prospective controlled study, Arab. J. Urol. 15 (2) (2017) 131-139.

[47] S.C. Esteves, M. Roque, A. Agarwal, Outcome of assisted reproductive technology in men with treated and untreated varicocele: systematic review and meta-analysis, Asian J. Androl. 18 (2) (2016) 254-258.

[48] L. Cheng, et al., Testicular cancer, Nat. Rev. Dis. Primers 4 (1) (2018) 29.

[49] P.F. Ray, et al., Genetic abnormalities leading to qualitative defects of sperm morphology or function, Clin. Genet. 91 (2) (2017) 217-232.

[50] M. Nigam, et al., Increasing incidence of testicular cancer in the United States and Europe between 1992 and 2009, World J. Urol. 33 (5) (2015) 623-631.

[51] S. Koul, et al., Characteristic promoter hypermethylation signatures in male germ cell tumors, Mol. Canc. 1 (2002) 8.

[52] C. Turnbull, et al., Variants near DMRT1, TERT and ATF7IP are associated with testicular germ cell cancer, Nat. Genet. 42 (7) (2010) 604-607.

[53] A.H. Dam, et al., Globozoospermia revisited, Hum. Reprod. Update 13 (1) (2007) 63-75.

[54] W. Vessey, et al., Reactive oxygen species in human semen: validation and qualification of a chemiluminescence assay, Fertil. Steril. 102 (6) (2014) 1576-1583 e4.

[55] H. Kobayashi, et al., Quality control of reactive oxygen species measurement by luminol-dependent chemiluminescence assay, J. Androl. 22 (4) (2001) 568-574.

[56] N. Desai, et al., Physiologic and pathologic levels of reactive oxygen species in neat semen of infertile men, Fertil. Steril. 92 (5) (2009) 1626-1631.

[57] A. Agarwal, G. Ahmad, R. Sharma, Reference values of reactive oxygen species in seminal ejaculates using chemiluminescence assay, J. Assist. Reprod. Genet. 32 (12) (2015) 1721-1729.

[58] A. Agarwal, et al., Determination of seminal oxidation-reduction potential (ORP) as an easy and cost-effective clinical marker of male infertility, Andrologia 50 (3) (2018) e12914.

[59] A. Agarwal, et al., MiOXSYS: a novel method of measuring oxidation reduction potential in semen and seminal plasma, Fertil. Steril. 106 (3) (2016) 566-573 e10.

[60] A. Agarwal, et al., Multi-center evaluation of oxidation-reduction potential by the MiOXSYS in males with abnormal semen, Asian J. Androl. 21 (6) (2019) 565-569.

[61] R. Mahfouz, et al., Diagnostic value of the total antioxidant capacity (TAC) in human seminal plasma, Fertil. Steril. 91 (3) (2009) 805-811.

[62] B.J.M. Mayorga-Torres, et al., Are oxidative stress markers associated with unexplained male infertility? Andrologia 49 (5) (2017).

[63] G. Aktan, et al., Mystery of idiopathic male infertility: is oxidative stress an actual risk? Fertil. Steril. 99 (5) (2013) 1211-1215.

[64] K. Mak, C.K. Kum, How to appraise a prognostic study, World J. Surg. 29 (5) (2005) $567-569$.

[65] B.W. Mol, et al., Characteristics of good diagnostic studies, Semin. Reprod. Med. 21 (1) (2003) 17-25

[66] S. Venkatesh, et al., Reactive oxygen species measurement in neat and washed semen: comparative analysis and its significance in male infertility assessment, Arch. Gynecol. Obstet. 283 (1) (2011) 121-126.

[67] R.A. Saleh, et al., Evaluation of nuclear DNA damage in spermatozoa from infertile men with varicocele, Fertil. Steril. 80 (6) (2003) 1431-1436.

[68] F.F. Pasqualotto, et al., Seminal oxidative stress in patients with chronic prostatitis, Urology 55 (6) (2000) 881-885.

[69] A. Vatannejad, et al., Evaluation of ROS-TAC score and DNA damage in fertile normozoospermic and infertile asthenozoospermic males, Urol. J. 14 (1) (2017) 2973-2978.

[70] F.F. Pasqualotto, et al., Poor semen quality and ROS-TAC scores in patients with idiopathic infertility, Urol. Int. 81 (3) (2008) 263-270.

[71] S.S. Allamaneni, et al., Characterization of oxidative stress status by evaluation of reactive oxygen species levels in whole semen and isolated spermatozoa, Fertil. Steril. 83 (3) (2005) 800-803.

[72] H. Elbardisi, et al., Predictive value of oxidative stress testing in semen for sperm DNA fragmentation assessed by sperm chromatin dispersion test, Andrology (2019), https://doi.org/10.1111/andr.12743.

[73] G. Balercia, et al., Placebo-controlled double-blind randomized trial on the use of Lcarnitine, L-acetylcarnitine, or combined L-carnitine and L-acetylcarnitine in men with idiopathic asthenozoospermia, Fertil. Steril. 84 (3) (2005) 662-671.

[74] G.M. Busetto, et al., Effect of metabolic and antioxidant supplementation on sperm parameters in oligo-astheno-teratozoospermia, with and without varicocele: a double-blind placebo-controlled study, Andrologia 50 (3) (2018).

[75] R. Jannatifar, et al., Effects of N-acetyl-cysteine supplementation on sperm quality, chromatin integrity and level of oxidative stress in infertile men, Reprod. Biol. Endocrinol. 17 (1) (2019) 24.

[76] M. Arafa, et al., Efficacy of antioxidant supplementation on conventional and advanced sperm function tests in patients with idiopathic male infertility, Antioxidants 9 (3) (2020).

[77] M.E. Hammadeh, et al., Reactive oxygen species, total antioxidant concentration of seminal plasma and their effect on sperm parameters and outcome of IVF/ICSI patients, Arch. Gynecol. Obstet. 277 (6) (2008) 515-526.

[78] L. Gambera, et al., Effects of antioxidant treatment on seminal parameters in patients undergoing in vitro fertilization, Arch. Ital. Urol. Androl. 91 (3) (2019).

[79] A. Zini, et al., Sperm DNA damage is associated with an increased risk of pregnancy loss after IVF and ICSI: systematic review and meta-analysis, Hum. Reprod. 23 (12) (2008) 2663-2668.

[80] L. Simon, et al., A systematic review and meta-analysis to determine the effect of sperm DNA damage on in vitro fertilization and intracytoplasmic sperm injection outcome, Asian J. Androl. 19 (1) (2017) 80-90. 